This is a pre-copyedited, author produced manuscript version of an article published in IMA Journal of Applied Mathematics following peer review. The version of record: Berezansky, L., Idels, L., \& Kipnis, M. (2011). Mathematical model of marine protected areas. IMA Journal of Applied Mathematics, 76(2), 312-325 is available online at: http://dx.doi.org/10.1093/imamat/hxq043. 


\title{
Mathematical Model of Marine Protected Areas
}

\author{
L. BEREZANSKY* \\ Department of Mathematics and Computer Science \\ Ben-Gurion University of Negev \\ Beer-Sheva 84105, Israel \\ email: brznsky@cs.bgu.ac.il \\ L. IDELS! \\ Department of Mathematics \\ Vancouver Island University (VIU) \\ 900 Fifth St. Nanaimo, BC, Canada V9S5J5 \\ email: lev.idels@viu.ca \\ M. KIPNIS $\ddagger$ \\ Department of Mathematics \\ Chelyabinsk State Pedagogical University \\ 69 Lenin Ave, Chelyabinsk 454080, \\ Russia \\ email: mmkipnis@gmail.com \\ Corresponding Author: Lev Idels
}

\footnotetext{
${ }^{*}$ Research supported in part by the Israeli Ministry of Absorption.

${ }^{\dagger}$ Research supported in part by a grant from VIU

${ }^{\ddagger}$ Research supported in part by Russian Foundation of Basic Research, grant No 0901-00896-a.
} 
Abstract. Considered two regions with a fish population that is dispersing between the two areas, and fishing takes place only in region 2 , with region 1 established as no-fishing zone. Marine protected areas (MPA) have been promoted as conservation and fishery management tools, and at present, there are over 1300 MPAs in the world. A new mathematical model of a MPA that reflects the complexity of the natural setting is presented. The resulting model of an age-structured fish population belongs to a class of nonlinear systems of differential equations with delay. New easily verifiable sufficient conditions for the existence, boundedness, permanence, and stability of the positive internal steady-state solutions are obtained. From the point of view of fishery managers, the existence of stable solutions is necessary for planning harvesting strategies and sustaining the fishing grounds. Numerical simulations illustrate qualitative behavior of the model, including stability switches.

Keywords - Marine Protected Areas, Fishery Models, Harvesting Models, System of Nonlinear Delay Differential Equations, Boundedness, Stability, Population Dynamics.

AMS Subject Classification: 34A34, 93A30, 37N25.

\section{Introduction}

Marine protected and marine reserves areas have been promoted as conservation and fishery management tools to hedge marine life and sustain ecosystems. The following definitions have emerged as commonly used terms [25]:

Marine Reserves are defined as areas completely protected in perpetuity from all extractive and destructive activities. Marine reserves are also known as "no-take areas" or "no-take/no-harm areas".

A "no-take" aquatic reserve means people are not permitted to fish by any method, destroy marine life, or collect dead or alive marine organisms, including empty shells. Reserves are a special category of marine protected areas.

Marine Protected Areas (MPA) are defined as areas designated to enhance conservation of marine resources through legal protections from disturbance, 
harm and/or fishing.

According to recent studies [1, 2], [10]-[14], [18, 24, 26, 27, 32] MPAs extend age structure of targeted species, sustain higher rate of reproduction, provide insurance against management failure, restrict fishing mortality, allow habitat to recover. Recent interest in modeling MPA has largely been driven by hope to fill some of the knowledge gaps and predict effectiveness of the reserves.

Two general approaches have been used to model MPA: mathematical models based on systems of ordinary differential equations (source-sink models) and empirical models based on the fitting programs.

Canonical logistic models of MPA $[8,7,13,14]$ are only capable of generating trivial equilibrium dynamics and unable to capture complex behavior often observed in nature; do not incorporate age structure and selective harvesting. In the existing patchy models [35] homogeneity of the patches, i.e. identical patches, is assumed; the latter undermined the actual MPA design.

The empirical models are based on the fitting programs such as spatially explicit Ecopath-Ecosim models [6] and produce a deterministic appraisal of current fisheries. The equilibrium model for predicting the efficacy of an MPA [33] is a step towards new ways to understand the benefits of MPA networks. Note that the effects of specific larval dispersal patterns on MPAs have been addressed via integro-difference equations in [17].

We introduce a MPA delayed model that incorporates age-structure, explicit spatial characteristics such as diffusion and/or migration; management criteria, such as the number of reserves, areas allocated to the protected and fishing zones, and selective harvesting strategies. The resulting model of an age-structured fish population belongs to a class of nonlinear systems of differential equations with delay, and presents interesting, non-trivial and unexpected mathematical problems that we will study.

The paper is organized as follows. In Section 2, we present biological motivation and description of the model. Biologically motivated conditions for the existence of positive steady state solutions are obtained in Section 3. The questions of the boundedness, interval bounds and persistence of positive solutions are studied in Section 4. By applying a new results obtained by authors in [19] and the Argument Principle techniques, sufficient conditions 
for stability of the model are obtained in Section 5. In the last Section via numerical simulations we illustrate our findings, discuss stability switches, and show that our model reflects the complexity of the natural setting.

\section{Delay Model of Marine Protected Areas}

To describe the ecological linkage between the reserve and fishing ground, we will consider two regions that have two areas $A_{1}$ and $A_{2}$. Assume that fishing takes place only in region 2, with region 1 established as a MPA or no-fishing zone, and fish is dispersing between the two areas. It is well-known $[7,11,31]$ that possible values of the system's control parameters are strongly related to biological and spatial characteristic, such as fish age and the size of the protected area. Let $t$ denotes a time and $a$ chronological age. We define the following functions: $u_{1}=u_{1}(t, a)$ is the age distribution of the fish population in the MPA; $u_{2}=u_{2}(t, a)$ is the age distribution of the fish population in the fishing area; $M_{1}(t, a)$ is the natural mortality rate in the MPA; $M_{2}(t, a)$ is the natural mortality. Let $D_{1}\left(A_{1}, a\right)$ be a net transfer rate, i.e. some net flow of adult fishes from the reserve, and $D_{2}\left(A_{2}, a\right)$ is the immigration rate from the fishing area to the reserve, say larval dispersion. We assume harvesting with a varying harvesting rate $H(t, a)$. Maintaining harvesting by restricting harvesting to fishes above a certain age or size (selective harvesting) is important and prevents population extinction.

Based on the conservation law $[5,34]$, we introduce the following model:

$$
\begin{aligned}
& \left(\frac{\partial}{\partial t}+\frac{\partial}{\partial a}\right) u_{1}(t, a)=-\left[M_{1}(t, a)+D_{1}\left(A_{1}, a\right)\right] u_{1}(t, a)+D_{2}\left(A_{2}, a\right) u_{2}(t, a), \\
& \left(\frac{\partial}{\partial t}+\frac{\partial}{\partial a}\right) u_{2}(t, a)= \\
& \quad-\left[M_{2}(t, a)+D_{2}\left(A_{2}, a\right)\right] u_{2}(t, a)+D_{1}\left(A_{1}, a\right) u_{1}(t, a)-H(t, a) u_{2}(t, a)
\end{aligned}
$$

with $u_{i}(0, a)=\omega_{i}(a) \geq 0(i=1,2)$, where $\omega_{i}(a)$ denotes initial conditions. If $\tau \geq 0$ is the maturation time, then the total matured population $x_{i}(t)$ at time $t$ is defined as

$$
x_{i}(t)=\int_{\tau}^{\infty} u_{i}(t, a) d a
$$

It is biologically reasonable to assume that only mature fish (with $a>\tau$ ) can reproduce and the reproduction rate depends on the mature population, 
i.e. $u_{i}(t, 0)=b_{i}\left(x_{i}(t)\right)$, where $b(\cdot)$ is the birth function; and $M_{i}(t, a)=m_{i}$, $D_{i}\left(A_{i}, a\right)=d_{i}(i=1,2)$ and $H(t, a)=h$.

As an example, we can choose Ricker's form [5] of the birth function:

$$
b_{i}\left(x_{i}\right)=x_{i} e^{-\alpha_{i} x_{i}}
$$

with positive constants $\alpha_{i}(i=1,2)$.

To tackle system (1), we used methods based on the technique of integration along characteristics [29, 30]. The resulting model for matured population becomes a system of DDE:

$$
\begin{aligned}
& \dot{x}_{1}=-m_{1} x_{1}-d_{1} x_{1}+d_{2} x_{2}+\gamma_{1} x_{1 \tau} e^{-\alpha_{1} x_{1 \tau}}, \\
& \dot{x}_{2}=-m_{2} x_{2}-d_{2} x_{2}+d_{1} x_{1}+\gamma_{2} x_{2 \tau} e^{-\alpha_{2} x_{2 \tau}}-h x_{2},
\end{aligned}
$$

and for $t \in[-\tau, 0]$

$$
x_{1}(t)=\varphi_{1}(t) \geq 0, \quad x_{2}(t)=\varphi_{2}(t) \geq 0,
$$

where $x_{i}=x_{i}(t)$ and $x_{i \tau}=x_{i}(t-\tau), \gamma_{i}=\gamma_{i}(\tau)=P_{i} e^{-\mu_{i} \tau}$, with nonnegative constants $\mu_{i}$ and $P_{i}(i=1,2)$.

Denote for $i=1,2$

$$
B_{i}(x)=\gamma_{i}(\tau) x e^{-\alpha_{i} x} .
$$

Functions $B_{i}(x)$ represent the growth rate, including a decreased survivorship $\gamma_{i}(\tau)$ over a longer incubation or maturation time. The general feature exhibited by functions $B_{i}(x)$ is that the population growth rate is positive when the population size is small, and then the birth rate function increases until it reaches the carrying capacity $B_{i}\left(1 / \alpha_{i}\right)=\frac{\gamma_{i}}{\alpha_{i} e}$ and then it decreases due to the crowding effect.

\section{Equilibrium Analysis}

To find all equilibria of system (3)-(4), we set

$$
\begin{aligned}
& m_{1} x_{1}+d_{1} x_{1}-d_{2} x_{2}-\gamma_{1} x_{1} e^{-\alpha_{1} x_{1}}=0, \\
& m_{2} x_{2}-d_{1} x_{1}+d_{2} x_{2}+h x_{2}-\gamma_{2} x_{2} e^{-\alpha_{2} x_{2}}=0 .
\end{aligned}
$$


From system (5) we have two curves:

$$
\begin{aligned}
& L_{1}: \quad x_{2}=\phi_{1}\left(x_{1}\right)=\frac{m_{1}+d_{1}-\gamma_{1} e^{-\alpha_{1} x_{1}}}{d_{2}} x_{1}, \\
& L_{2}: \quad x_{1}=\phi_{2}\left(x_{2}\right)=\frac{m_{2}+d_{2}+h-\gamma_{2} e^{-\alpha_{2} x_{2}}}{d_{1}} x_{2} .
\end{aligned}
$$

Apart from the zero solution, system (6) has a nontrivial positive solution $\left(\bar{x}_{1}, \bar{x}_{2}\right)$.

Theorem 3.1 System (3) has a unique internal positive equilibrium if the following conditions hold:

(i) $m_{1}+d_{1} \leq \gamma_{1}$ and (ii) $m_{2}+d_{2}+h \leq \gamma_{2}$.

Proof. To prove the existence of a nontrivial equilibrium, firstly note that curves $L_{1}$ and $L_{2}$ have asymptotes

$$
x_{2}=\frac{m_{1}+d_{1}}{d_{2}} x_{1}
$$

and

$$
x_{2}=\frac{d_{1}}{m_{2}+d_{2}+h} x_{1}
$$

correspondingly. Clearly,

$$
\frac{m_{1}+d_{1}}{d_{2}}>\frac{d_{1}}{m_{2}+d_{2}+H}
$$

thus, for sufficiently large $x_{1}$, points on the curve $L_{1}$ lie above the corresponding points of the curve $L_{2}$. On the other hand, in the neighborhood of the origin, conditions (i)-(ii) guarantee that points on the curve $L_{2}$ lie above points of the curve $L_{1}$. Therefore a positive internal equilibrium of system (3)-(4) exists.

To prove that this equilibrium is a unique point, firstly, we note that from system (6)

$$
\begin{aligned}
& L_{1}: \quad \frac{d x_{2}}{d x_{1}}=\frac{x_{2}}{x_{1}}+\frac{\gamma_{1}}{d_{2}} \alpha_{1} x_{1} \exp \left(-\alpha_{1} x_{1}\right)>\frac{x_{2}}{x_{1}}, \\
& L_{2}: \quad \frac{d x_{1}}{d x_{2}}=\frac{x_{1}}{x_{2}}+\frac{\gamma_{2}}{d_{1}} \alpha_{2} x_{2} \exp \left(-\alpha_{2} x_{2}\right)>\frac{x_{1}}{x_{2}},
\end{aligned}
$$


since $x_{1}>0$ on $L_{1}$ and $x_{2}>0$ on $L_{2}$. Let $\theta$ be a polar angle of the point on the curve $L_{1}$ (with $O x_{1}$ as a polar axis),

$$
\theta=\arctan \frac{x_{2}}{x_{1}}=\arctan \frac{\phi_{1}}{x_{1}} .
$$

If $x_{1}$ moves from 0 to $\infty$, then

$$
\frac{d \theta}{d x_{1}}=\frac{1}{1+\left(\frac{x_{2}}{x_{1}}\right)^{2}} \frac{d}{d x_{1}} \frac{\phi_{1}}{x_{1}}=\frac{x_{1}}{x_{1}^{2}+x_{2}^{2}}\left(\frac{d \phi_{1}}{d x_{1}}-\frac{\phi_{1}}{x_{1}}\right) .
$$

The latter equality and inequalities (7) guarantee $\frac{d \theta}{d x_{1}}>0$, thus $\theta\left(x_{1}\right)$ is a monotone increasing function with

$$
\theta_{0}<\theta\left(x_{1}\right)<\arctan \frac{m_{1}+d_{1}}{d_{2}}
$$

Similarly, let $\vartheta$ be a polar angle of the point on the curve $L_{2}$. If $x_{2}$ moves from 0 to $\infty$, then $\vartheta\left(x_{2}\right)$ is a monotone decreasing function, with

$$
\arctan \frac{d_{1}}{m_{2}+d_{2}+h}<\vartheta\left(x_{2}\right)<\vartheta_{0} .
$$

At the equilibrium point $\theta=\vartheta$, increase of the function $\theta$ and decrease of the function $\vartheta$ guarantee the uniqueness of a nontrivial equilibrium.

Let none of the conditions (i)-(ii) hold. Then for any $x_{1}>0$ and $x_{2}>0$

$$
\begin{aligned}
& \arctan \frac{m_{1}+d_{1}}{d_{2}}>\theta \geq \theta_{0}=\arctan \frac{m_{1}+d_{1}-\gamma_{1}}{d_{2}}> \\
& \vartheta_{0}=\arctan \frac{d_{1}}{m_{2}+d_{2}+h-\gamma_{2}} \geq \vartheta>\arctan \frac{d_{1}}{m_{2}+d_{2}+h} .
\end{aligned}
$$

Therefore the equality $\theta=\vartheta$ is impossible, and the positive equilibrium does not exist. Theorem 3.1 is proved.

Remark 3.1 Without migration $\left(d_{1}=d_{2}=0\right)$, the delay differential model (3) is a direct extension of the well-known Nicholson models [5]. Nonmigration system

$$
\begin{aligned}
& \dot{x}_{1}=-m_{1} x_{1}+\gamma_{1} x_{1}(t-\tau) \exp \left(-\alpha_{1} x_{1}(t-\tau)\right), \\
& \dot{x}_{2}=-\left(m_{2}+h\right) x_{2}+\gamma_{2} x_{2}(t-\tau) \exp \left(-\alpha_{2} x_{2}(t-\tau)\right)
\end{aligned}
$$


has a positive equilibrium

$$
\left(\frac{1}{\alpha_{1}} \ln \frac{\gamma_{1}}{m_{1}}, \frac{1}{\alpha_{2}} \ln \frac{\gamma_{2}}{m_{2}+h}\right)
$$

provided $m_{1}<\gamma_{1}$ and $m_{2}+h<\gamma_{2}$.

Remark 3.2 According to [30], the equilibrium point $\bar{x}_{1}=\bar{x}_{2}=\bar{x}$ is called a homogeneous equilibrium. In order to obtain the condition of existence of such an equilibrium for model (3), we shall use system (5).

Proposition 3.1 If

$$
\frac{\gamma_{1}}{m_{1}+d_{1}-d_{2}}=\left(\frac{\gamma_{2}}{m_{2}+d_{2}-d_{1}+h}\right)^{\frac{\alpha_{1}}{\alpha_{2}}},
$$

then a homogeneous equilibrium of system (3) exists, and

$$
\bar{x}=\frac{1}{\alpha_{1}} \ln \frac{\gamma_{1}}{m_{1}+d_{1}} .
$$

\section{Boundedness of the Solutions}

Consider system (3) with initial conditions (4) in a vector form

$$
\frac{d x}{d t}=A x+f(x(t-\tau)),
$$

where

$$
\begin{gathered}
A=\left[\begin{array}{cc}
-\left(m_{1}+d_{1}\right) & d_{2} \\
d_{1} & -\left(m_{2}+d_{2}+h\right)
\end{array}\right], \\
x(t)=\left[\begin{array}{l}
x_{1}(t) \\
x_{2}(t)
\end{array}\right], \quad f=\left[\begin{array}{l}
f_{1} \\
f_{2}
\end{array}\right]
\end{gathered}
$$

with

$$
f_{i}=\gamma_{i} x_{i}(t-\tau) \exp \left(-\alpha_{i} x_{i}(t-\tau)\right), \quad(i=1,2)
$$

We denote

$$
a=\frac{2 \max \left(\gamma_{1}, \gamma_{2}\right)}{\min \left(m_{1}, m_{2}\right) \min \left(\alpha_{1}, \alpha_{2}\right)} .
$$


Theorem 4.1 Any solution of system (10) with nonnegative initial functions and positive initial conditions ultimately enters the square region

$$
K=\left\{0<x_{1}(t) \leq a, 0<x_{2}(t) \leq a\right\},
$$

where $a$ is defined by (11), and remains in it.

Proof. Note that from standard differential equation theory [4], system (3) has solution $x_{1}(t)>0, x_{2}(t)>0$ for $t>0$, provided that $x_{1}(0)>0, x_{2}(0)>0$ and

$$
x_{1}(t)=\varphi_{1}(t) \geq 0, \quad x_{2}(t)=\varphi_{2}(t) \geq 0,
$$

for $t \in[-\tau, 0)$.

Let $z=x_{1}+x_{2}$, then from system (10) we have

$$
\frac{d z}{d t}=-m_{1} x_{1}-m_{2} x_{2}-h x_{2}+f_{1}\left(x_{1}(t-\tau)\right)+f_{2}\left(x_{2}(t-\tau)\right) .
$$

For $0<\epsilon<\min \left(m_{1}, m_{2}\right)$ we have

$$
\begin{array}{r}
\frac{d z}{d t}+\epsilon z=-\left(m_{1}-\epsilon\right) x_{1}-\left(m_{2}-\epsilon\right) x_{2}-h x_{2}+ \\
f_{1}\left(x_{1}(t-\tau)\right)+f_{2}\left(x_{2}(t-\tau)\right)< \\
f_{1}\left(x_{1}(t-\tau)\right)+f_{2}\left(x_{2}(t-\tau)\right) \leq \max \left\{f_{1}, f_{2}\right\} \leq \max \left\{\frac{\gamma_{1}}{\alpha_{1}}, \frac{\gamma_{2}}{\alpha_{2}}\right\}=W .
\end{array}
$$

Therefore,

or

$$
\frac{d z}{d t}+\epsilon z<W
$$

$$
z(t)<\frac{W}{\epsilon}+\left(z(0)+\frac{W}{\epsilon}\right) e^{-\epsilon t} .
$$

Thus all solutions of system (3) along with system (10) are bounded.

We note that due to usual arguments (see for example [16]) the local solution is not a global solution if there exists $t_{1}>0$ such that lim $\sup _{t \rightarrow t_{1}-}|x(t)|=\infty$. But all solutions of system (10) are positive and bounded functions, hence every local solution is also a global solution.

As $x_{1}(t)>0, x_{2}(t)>0$ we have from (12) that there exists $t_{0}$, such that $x_{i}(t)<2 W / \epsilon<a(i=1,2)$ for $t_{0} \leq t<\infty$. Theorem 4.1 is proved. 


\section{$5 \quad$ Stability Analysis}

Note that the general theory of scalar nonlinear DDEs has been well studied [16], whereas the theory of the systems of DDEs, especially nonlinear systems, is a relatively new research endeavour [9, 28, 35]. This raises the issue as to whether the techniques developed for scalar equations can be further extended to the systems of DDEs.

For a scalar Nicholson delay differential equation

$$
\dot{x}=-m x(t)+\gamma x(t-\tau) e^{-a x(t-\tau)}
$$

the delay-dependent asymptotic stability condition was obtained in [15] (see also [3]):

$$
0<\left(e^{m \tau}-1\right)\left(\frac{\gamma}{m}-1\right)<1
$$

There is a critical value of the time delay that switches stability to of a limit-cycle type fluctuations. According to [23], the period of the cycles is set mainly by the delay $\tau$ and mortality rate $m$. High values of $\gamma \tau$ and $m \tau$ will give large amplitude cycles.

A non-migration model (9) is a decoupled Nicholson system, therefore the conditions

$$
0<\left(e^{m_{1} \tau}-1\right)\left(\frac{\gamma_{1}}{m_{1}}-1\right)<1
$$

and

$$
0<\left(e^{\left(m_{2}+h\right) \tau}-1\right)\left(\frac{\gamma_{2}}{m_{2}+h}-1\right)<1
$$

guarantee the global asymptotical stability of the steady state solution of system (9) for all $t>0$ and $\tau \geq 0$.

Consider a nonlinear system

$$
\begin{aligned}
& \frac{d x}{d t}=A x(t)+F\left(t, x\left(t-\tau_{1}\right), \ldots, x\left(t-\tau_{k}\right)\right), \quad t>0, \\
& 0<\tau_{1} \leq \tau_{2} \leq \ldots \leq \tau_{k}, \quad x(t)=\phi(t), \quad t \in\left[-\tau_{k}, 0\right],
\end{aligned}
$$

where $x:\left[-\tau_{k}, \infty\right] \rightarrow \mathbb{R}^{n}, A \in \mathbb{R}^{n \times n}$. here $F: \mathbb{R}_{+} \times \mathbb{R}^{n \times k} \rightarrow \mathbb{R}^{n}$ nonlinear and continuous vector function. We shall use $|\cdot|,|| \cdot||$ to denote norms in $\mathbb{R}^{n}$ and $\mathbb{R}^{n \times n}$.

Let $\mu(A): \mathbb{R}^{n \times n} \rightarrow \mathbb{R}$ be a function such that

$$
\left\|e^{A t}\right\| \leq e^{\mu(A) t}
$$

In [19] we proved the following result: 
Theorem 5.1 Suppose that there exist the nonnegative sequence $\xi_{i}(1 \leq$ $i \leq k)$, such that for every $u_{i} \in \mathbb{R}^{n}$ and $t \in \mathbb{R}$ the inequalities

$$
\begin{gathered}
\left|F\left(t, u_{1}, u_{2}, \ldots, u_{k}\right)\right| \leq \sum_{i=1}^{k} \xi_{i}\left|u_{i}\right| \\
-\mu(A)>\sum_{i=1}^{k} \xi_{i}
\end{gathered}
$$

hold. Then there are positive constants $\sigma$ and $C$ such that every solution $x(t)$ of the system (13) satisfies the inequality

$$
|x(t)| \leq C e^{-\sigma t} \max _{-\tau_{k} \leq t \leq 0}|\phi(t)| .
$$

Based on this theorem, we also proved

\section{Theorem 5.2 If}

$$
\min \left(m_{1}, m_{2}+h\right) \geq \max \left(\gamma_{1}, \gamma_{2}\right)
$$

then zero solution of the system (10) is globally asymptotically stable.

Let

$$
z(t)=\left[\begin{array}{l}
x_{1}(t)-\bar{x}_{1} \\
x_{2}(t)-\bar{x}_{2}
\end{array}\right] .
$$

Then a linearization of system (10) has the following vector form

$$
\frac{d z}{d t}=A z(t)-G(\tau) z(t-\tau)
$$

where

$$
G(\tau)=\left[\begin{array}{cc}
g_{1}(\tau) & 0 \\
0 & g_{2}(\tau)
\end{array}\right]
$$

with

$$
g_{i}(\tau)=\gamma_{i}(\tau) \exp \left(-\alpha_{i} \bar{x}_{i}\right)\left(\alpha_{i} \bar{x}_{i}-1\right), \quad(i=1,2)
$$

The stability of a nontrivial equilibrium of system (15) is determined by the roots of the quasi-polynomial characteristic equation

$$
\lambda^{2}+q \lambda+s+\left[c(\tau) \lambda+d(\tau)+r(\tau) e^{-\lambda \tau}\right] e^{-\lambda \tau}=0,
$$


where

$$
\begin{aligned}
& q=m_{1}+d_{1}+m_{2}+d_{2}+h, \\
& s=\left(m_{1}+d_{1}\right)\left(m_{2}+d_{2}+h\right)-d_{1} d_{2}, \\
& c(\tau)=g_{1}(\tau)+g_{2}(\tau), \\
& r(\tau)=g_{1}(\tau) g_{2}(\tau), \\
& d(\tau)=\left(m_{1}+d_{1}\right) g_{2}(\tau)+\left(m_{2}+d_{2}+h\right) g_{1}(\tau) .
\end{aligned}
$$

Note that $q>0, s>0$.

We assume that

$$
\gamma_{1}(\tau)>\left(m_{1}+d_{1}\right) e, \quad \gamma_{2}(\tau)>\left(m_{2}+d_{2}+h\right) e .
$$

Clearly, conditions (19) imply the inequalities $\alpha_{i} \bar{x}_{i}>1(i=1,2)$, thus all functions $c(\tau), r(\tau)$ and $d(\tau)$ are positive functions.

Denote

$$
F(\lambda)=\lambda^{2}+q \lambda+s+\left[c(\tau) \lambda+d(\tau)+r(\tau) e^{-\lambda \tau}\right] e^{-\lambda \tau} .
$$

If for any root $\lambda$ of equation (17) we have $\operatorname{Re}(\lambda)<0$, then system (15) is asymptotically stable. If there exists a root $\lambda$, such that $\operatorname{Re}(\lambda)>0$, then the system is unstable [21].

To investigate stability of system (15) we shall use the argument principle [20]. Consider a contour $K$ of a complex plane consisting of a segment $I_{R}: \lambda=-i \omega(-R \leq \omega \leq R)$ and semicircle $C_{R}: \lambda=R e^{i \omega}$ $(-\pi / 2 \leq \omega \leq \pi / 2)$

By the principle of argument [20], if $\lambda$ moves along $K$, then $\Delta \operatorname{Arg} F(\lambda)=$ $2 \pi N$, where $N$ is a number of roots of equation (17) inside the contour $K$.

Hence, the necessary and sufficient condition for asymptotic stability of system $(15)$ is

$$
\Delta \operatorname{Arg} F(\lambda)=0,
$$

when $\lambda$ moves along $K$ with sufficiently large $R$. We call the curve $F(\lambda)$ a hodograph. Since $\lambda^{2}$ is the main term in $F(\lambda)$, we have $\Delta \operatorname{Arg} F(\lambda) \rightarrow 2 \pi$, while $\lambda$ moves along the curve $C_{R}$ with $R \rightarrow \infty$. Hence, for the asymptotic stability of system (15) it is necessary and sufficient to have the condition

$$
\Delta \operatorname{Arg} F(\lambda)=-2 \pi
$$

where $\lambda$ moves along $I_{R}$ with $R \rightarrow \infty$. By symmetry an equivalent form of the latter condition is written:

$$
\Delta \operatorname{Arg} F(-i \omega)=-\pi \quad(0 \leq \omega<\infty)
$$


Denote $u(\omega)=\operatorname{Re} F(-i \omega)$ and $v(\omega)=\operatorname{Im} F(-i \omega)$. Then

$$
\begin{aligned}
& u(\omega)=-\omega^{2}+s+c \omega \sin \omega \tau+d(\tau) \cos \omega \tau+r(\tau) \cos 2 \omega \tau, \\
& v(\omega)=-q \omega-c \omega \cos \omega \tau+d(\tau) \sin \omega \tau+r(\tau) \sin 2 \omega \tau .
\end{aligned}
$$

Finally, since $\operatorname{Arg} F(0)=0$, we have the following stability diagnosis procedure for system (15). Firstly, draw curve (21) for $0 \leq \omega<\infty$. If $\operatorname{Arg}(u(\omega)+i v(\omega)) \rightarrow-\pi$ with $\omega \rightarrow \infty$, then system (15) is asymptotically stable, otherwise it is unstable. For the qualitative estimations of the stability domain we shall provide the explicit sufficient stability conditions in the space of the parameters.

Theorem 5.3 If

$$
q>c+\tau d(\tau)+2 \tau r(\tau)
$$

then $\operatorname{Re}(\lambda)<0$ for any root $\lambda$ of quasi-polynomial (20).

Proof. Condition (22) implies $v(\omega)<0$ when $\omega>0$. Hence the value of $\operatorname{Arg} F(-i \omega)$ varies continuously from 0 to $(-\pi)$ while $\omega$ moves from 0 to $\infty$. Theorem 5.3 is proved.

\section{Corollary 5.1 If}

$$
q>c+\tau d(\tau)+2 \tau r(\tau)
$$

then system (15) is asymptotically stable.

Example 5.1 To illustrate trajectories of the nonlinear system (3) we choose the following parameters

$$
\begin{aligned}
& m_{1}=0.2, m_{2}=0.5, d_{1}=d_{2}=1, h=0.7, \\
& \gamma_{1}(\tau)=14, \gamma_{2}(\tau)=7, \alpha_{1}=0.16, \alpha_{2}=0.9 .
\end{aligned}
$$

System (15) has a nonzero equilibrium $\bar{x}_{1}=18.337, \bar{x}_{2}=8.349$. Data set (23) and equalities (16),(18) yield

$$
q=3.400, s=1.640, c=1.465, r=0.036, d=1.783 .
$$

In Fig.1a the delay $\tau=1$ is sufficiently small to satisfy the inequality (22) of Theorem 5.3 and Corollary 5.1, thus the nonzero equilibrium point is asymptotically stable. Fig.1b illustrates instability of the equilibrium point for the 
delay value $\tau=3$. Trajectories in Fig. 1 were calculated by the MATLAB program DDE23.

"Delay-independent" stability condition is constructed in the following theorem.

\section{Theorem 5.4 If}

$$
s>1+c+d(\tau)+r(\tau) \text { and } q>c+d(\tau)+r(\tau)
$$

then $\operatorname{Re}(\lambda)<0$ for any root $\lambda$ of quasi-polynomial (20).

Proof. First condition of (25) implies $u(\omega)>0$ when $0 \leq \omega \leq 1$. Second condition of (25) guaranteed the inequality $v(\omega)<0$ when $\omega>1$. Hence (see $(21)$ ) the hodograph $F(-i \omega)$ avoids the quarter $u<0, v>0$ of the $(u, v)$-plane. Therefore $\Delta \operatorname{Arg} F(-i \omega)=-\pi$ while $\omega$ moves from 0 to $\infty$. Theorem 5.4 is proved.
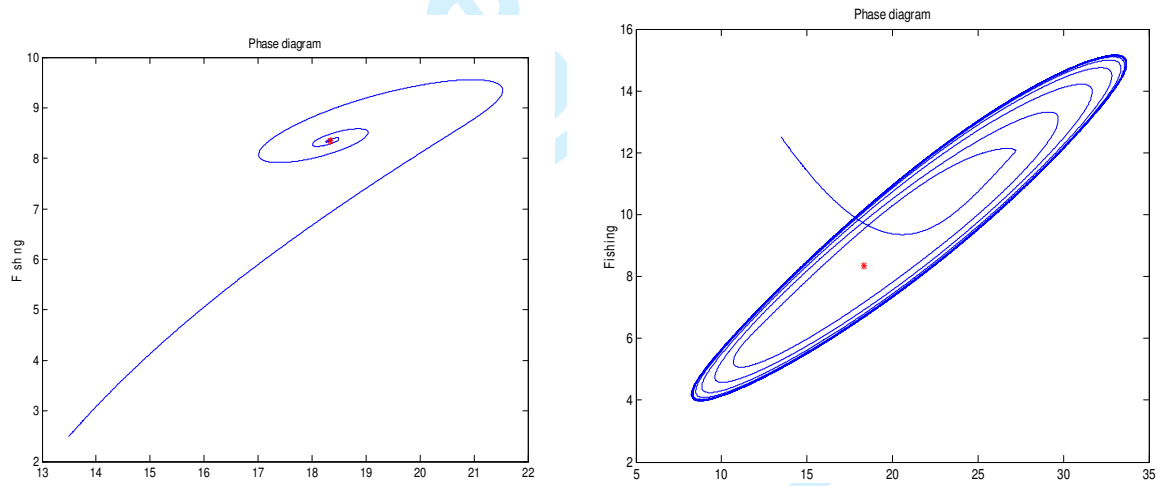

Figure 1: Trajectories of nonlinear system (3) with the parameters given by data set (23): a) $\tau=1$, b) $\tau=3$.

Consider now instability condition for system (15).

\section{Theorem 5.5 If}

$$
\frac{\pi(q+c)}{2(d(\tau)+2 r(\tau))}<\tau<\frac{\pi}{4(c+\sqrt{d(\tau)+s+r(\tau)})}
$$


then system (15) is unstable.

Proof. Consider $\omega \tau \leq \frac{\pi}{4}$ in hodograph (21). The left-hand side of inequality (26) implies $\pi(q+c) \omega<2 \tau d(\tau) \omega+4 \tau r(\tau) \omega$, hence system (21) with $\omega \tau \leq \frac{\pi}{4}$ yields

$$
v(\omega)>-q \omega-c \omega+d(\tau) \frac{2}{\pi} \omega \tau+r(\tau) \frac{4}{\pi} \omega \tau>0 .
$$

Let $\omega \tau>\frac{\pi}{4}$. The right-hand side of (26) implies

$$
\omega>\frac{\pi}{4 \tau}>c+\sqrt{d(\tau)+s+r(\tau)} .
$$

Therefore

$$
\omega^{2}-c \omega>\omega \sqrt{d(\tau)+s+r(\tau)}>c \sqrt{d(\tau)+s+r(\tau)}+d(\tau)+s+r(\tau),
$$

hence (see $(21)) u(\omega)<0$. Thus with any value of $\omega \in(0, \infty)$ hodograph (21) avoids the domain $u>0, v<0$.

Finally, $\Delta \operatorname{Arg} F(-i \omega)=\operatorname{Arg} F(-i \infty)-\operatorname{Arg} F(0)=\pi$. Theorem 5.5 is proved.

Remark 5.1 A gap between the left- and right-hand side of (26) is nonempty, when the value of $d(\tau)$ is sufficiently large compare with $q, c, r(\tau), s$. The latter is consistent with inequalities (25). For example, if $q=0.3, c=$ $0.3, r(\tau)=0.1, s=0.1, d=3.8$, constraints (25) are satisfied, therefore system (15) is unstable by Theorem 5.5.

\section{Discussion}

In this paper, we made an attempt toward modeling and analyzing a simple marine protected areas model for a fish population. The novel aspect of our model is the incorporation of age structure, migration and selective harvesting. The resulting model belongs to a class of nonlinear delay differential equations. Specifically, we obtained necessary and sufficient conditions for the existence of steady equilibrium points. We also proved that all solutions are nonnegative and bounded for every set of the positive initial functions and initial conditions. For qualitative estimations of the stability domain in the space of parameters along with the stability diagnosis, explicit sufficient 
stability conditions are obtained. To find the regions of local stability or instability of the system, we used the Argument Principle for a direct analysis of the characteristic equation. We would like to point out that assumptions and constraints for all theorems in this paper are biologically motivated.

Our most interesting results are Theorems 5.3 and 5.5 and its implications. Although all theorems are established for model (3), most of its important biological implications should remain true for more advanced models of MPA, including diffusion models. Numerical simulations illustrate existence of stability switches in the model. It follows from our findings that introduction of protected areas could save a fish population even under excessive harvesting. There are many assumptions and simplifications in our model that are open to objections. Some of the modifications in this model could change our results; others might not, the latter leaves some open problems for future work. 


\section{References}

[1] AMI, D., CARTIGNY, P. RAPAPORT, A. (2005) Can marine protected areas enhance both economical and biological situations? C.R. Biologies, 328, 357-366

[2] ARMSTRONG, C., (2007) A note on the ecological-economic modelling of marine reserves in fisheries, Ecol. Economics. 62 , 242-250

[3] L. BEREZANSKY, B., BRAVERMAN, E., IDELS, L. (2009) Nicholson Blowflies Differential Equations Revisited: Main Results and Open Problems, Applied Math Modelling, 34 (6) 1405-17

[4] BELLMAN, R., COOKE K.,(1963) Differential-Difference Equations. Mathematics in Science and Engineering, Volume 28

[5] BRAUER, F., CASTILlO-CHAVEZ, C. (2001) Mathematical Models in Population Biology and Epidemiology, Springer-Verlag

[6] CHRistensen, V., WALTERS, C. (2004) Ecopath with Ecosim: methods, capabilities and limitations, Eco. Modelling, 172 , 109-139

[7] DOYEN, L., BENE, C. (2003) Sustainability of fisheries through marine reserves: a robust modeling analysis, J. of Env. Management, 69 , 1-13

[8] DUBEY, B., CHANDRA, P., SINHA, P. (2000) A model for fishery resource with reserve area, Nonlinear Analysis: Real World Appl. 4, $3625-637$

[9] FABIANO, R.,TURI, J. (2006) Stability for retarded delay equations with multiple delays, Decision and Control, 45th IEEE Conference, pp. $4712-4716$

[10] GELL, F., ROBERTS, C. (2003) Benefits beyond boundaries: the fishery effects of marine reserves, Trends in Ecology and Evolution 18(9) 448-455

[11] GERBER, L., KAREIVA, P., BASCOMPTE, J. (2002) The influence of life history attributes and fishing pressure on the efficacy of marine reserves, Biol. Conservation 106 , 11-18 
[12] GERBER, L., et al. (2003) Population models for marine reserve design: a retrospective and prospective synthesis, Ecol. Appl. 13(1), 547-564

[13] GRAFton R., KOMPAS, T., LINDEnMAYER, D. (2005) Marine reserves with ecological uncertainty, Bull. of Math. Bio. 67 , 957-971

[14] GUENETTE, S., PITCHER T. (1999) An age-structured model showing the benefits of marine reserves in controlling overexploitation, Fisheries Research 39, 295-303

[15] GYÖRY, I., LADAS G. (1991) Oscillation Theory of Delay Differential Equations, Clarendon Press, Oxford

[16] HALE, J., LUNEL, S. (1993) Introduction to Functional Differential Equations. Appl. Math. Sci. Springer-Verlag, NY, vol. 99

[17] HASTINGS, A., BOTSFORD, L. (2003) Comparing designs of marine reserves for fisheries and for biodiversity, Ecol. Appl. 13(1) 865-870

[18] HAUSER, C., COOCH, E., LEBERTON, J. (2006) Control of structured populations by harvest, Eco. Model., 196 462-470

[19] IDELS, L., KIPNIS, M. (2008), Stability criteria for a nonautonomous nonlinear system with delay, Applied Mathematical Modelling, 33 (5), 2293-2297

[20] KNOPP, K. (1996) Theory of Functions, Part II. New York: Dover,

[21] KUANG, Y. (1993) Delay Differential Equations With Applications In Population Dynamics, N.Y. Academic Press

[22] LI, J., MA, Z. (2004) Stability switches in a class of characteristic equations with delay dependent parameters, Nonlin. Anal.: Real World Appl. 5, 389-408

[23] LI, W., RUAN, S., WANG, Z. (2007) On the diffusive Nicholson's blowflies equation with nonlocal delay, J. Nonlinear Sci 17505-525

[24] LOCKWOOD, D., HASTINGS, A., BOTSFORD, L. (2002) The effects of dispersal patterns on marine reserves: Does the tail wag the dog?, Theor.Pop. Biology 61, 297-309 
[25] LUBCHENKO, J., et al (2003) Plugging a hole in the ocean: the emerging science of marine reserves, Ecological Applications 13(1) Suppl., 3-7

[26] NOWLIS, S., ROBERTS, C. (1999) Fisheries Benefits and optimal design of marine reserves, Fisheries Bull. 97(3) 604-616

[27] PITCHFORD, J., CODLING, E., PSARRA, D. (2007) Uncertainty and sustainability in fisheries and the benefit of MPA, Ecol. Model., 207 286-292

[28] RUAN, S. (2009) On Nonlinear Dynamics of Predator-Prey Models with Discrete Delay, Math. Model. Nat. Phenom. Vol. 4, No. 2

[29] RUAN, S., G. WOLKOWICZ G., WU, J. (2003) Dynamical Systems and Their Applications in Biology AMS - FI,

[30] SMITH, H., THIEME, H. (1991) Convergence for strongly orderpreserving semiflows. SIAM J. Math. Anal. 22, no. 4, 1081-1101

[31] SOBEL, J., DAHLGREN, G. (2004) Marine Reserves: A guide to science, design, and use, Island Press, Washington,

[32] SUMAILA, U., et al (2000) Addressing ecosystem effects of fishing using marine protected areas, ICES J. of Marine Sci. 57, 752-760

[33] WALTERS, C., HILBORN, R., PARRISH, R. (2007) Equilibrium model for predicting the efficacy of marine protected areas in coastal environment, Can. J. Fish Aquat. Sci., 64, 1009-18.

[34] WEBB, G. (1985) Theory of Nonlinear Age-dependent Population Dynamics, Marcel Dekker, NY

[35] XU, D. (2005) Global dynamics and Hopf bifurcation of a structured population model, Nonlinear Anal.: real world Appl., 6, 461-476 